\title{
LOS ANTECEDENTES DE LA CREACIÓN DEL CENTRO DE INVESTIGACIONES GEOFÍSICAS (CIGEFI) DE LA UNIVERSIDAD DE COSTA RICA
}

\author{
Walter Fernández \\ Laboratorio de Investigaciones Atmosféricas y Planetarias, Escuela de Física \\ y Centro de Investigaciones Geofísicas, Universidad de Costa Rica, \\ San José, Costa Rica \\ Correo electónico: wfer@ cariari.ucr.ac.cr
}

\section{INTRODUCCIÓN}

Las ciencias geofísicas estudian los procesos que ocurren en los interiores, superficies y atmósferas de los planetas, así como sus interacciones con el espacio. Esto incluye varias disciplinas. Por ejemplo, la Unión Geofísica Americana tiene secciones en los siguientes campos: 1) Geodesia, 2) Sismología, 3) Tectonofísica, 4) Vulcanología, Geoquímica y Petrología, 5) Geomagnetismo y Paleomagnetismo, 6) Hidrología, 7) Ciencias del Océano, 8) Ciencias de la Atmósfera, 9) Aeronomía y Física del espacio y 10) Planetología. Muchos de estos campos tienen gran relevancia en aspectos tales como el cambio global, la reducción de los efectos de las catástofes naturales, las fuentes de energía no convencionales y los recursos naturales.

Al regresar el autor de este artículo al país e incorporarse a la Universidad de Costa Rica en 1974, se encontró con que las modificaciones propuestas por el Tercer Congreso Universitario en 1973 favorecían la creación de un centro o instituto de carácter interdisciplinario, que se dedicara a realizar investigaciones en ciencias geofísicas. Había considerado varios ejemplos: los institutos de Geofísica y Física Planetaria de algunas universidades de los Estados Unidos (principalmente en California), el Instituto de Geofísica de la Universidad de Alaska y el Instituto de Geofísica de la UNAM (México). Conversé al respecto con el profesor German Leandro, un excompañero de estudio que después de graduarse en Física, había realizado estudios de Geofísica (con énfasis en prospección) en México. El profesor Leandro se interesó mucho en el proyecto y lo apoyó en todo. También le comuniqué esta idea al Ing. Elliott Coen, profesor y jefe de laboratorios de la Escuela de Física, quien se interesó mucho por esa iniciativa. Posteriormente, hablé al respecto con la M.Sc. Luz Soltero, profesora de la Escuela de Física, quien con gran amabilidad participó en la promoción del proyecto y acompañó al profesor German Leandro y al autor a varias reuniones que sostuvieron con autoridades universitarias sobre la creación de dicho 
centro o instituto. De estas conversaciones, las principales fueron con el Dr. Guillermo Chaverri, Vicerrector de Investigación de aquel entonces, el Dr. Fernando Durán, Coordinador del Area de Ciencias y Decano de la Facultad de Ciencias, el Dr. César Dóndoli, Director de la Escuela Centroamericana de Geología, y el M.Sc. Neville Clark, Director de la Escuela de Física.

\section{PRESENTACIÓN DE LA PROPUESTA DE LA CREACIÓN DEL CENTRO}

Los profesores Walter Fernández, German Leandro y Luz Soltero le enviaron al señor Vicerrector de Investigación, Dr. Guillermo Chaverri, un proyecto para la creación del "Centro de Geofísica y Física Planetaria”, solicitándole su aprobación. El señor Vicerrector les contestó el 5 de diciembre de 1974 (oficio VI-13974) diciendo: "Me refiero a los documentos que ustedes me enviaron relacionados con la creación de una División adscrita al Instituto de Ciencias, denominada "Geofísica y Física Planetaria". Considero de gran importancia este proyecto, por lo que tiene la aprobación en principio, de esta Vicerrectoría ya que el Consejo Universitario será quien dará la última palabra sobre el asunto. Deberán enviar el proyecto antes mencionado, a conocimiento del Consejo de Area de Ciencias con el fin de que se pronuncie al respecto. Cuando haya obtenido el trámite respectivo, deberá poligrafiarse y enviarse 10 copias a esta Vicerrectoría..." El 17 de diciembre de 1974, los profesores Walter Fernández y German Leandro le enviaron una carta al Dr. Fernando Durán (en su calidad de Coordinador del Area de Ciencias), con copia al Dr. Guillermo Chaverri, en la que se dice: "Siguiendo instrucciones del Dr. Guillermo Chaverri, Vicerrector de Investigación, le solicitamos muy atentamente someter a conocimiento del Consejo de Area de Ciencias, el proyecto para la creación del Centro de Geofísica y Física Planetaria, con el fin de que dicho Consejo se pronuncie al respecto..." El Dr. César Dóndoli, Director de la Escuela Centroamericana de Geología, sugirió varias modificaciones a las cuales el autor de este artículo les hizo varios comentarios en una carta que le envió al Dr. Dóndoli el 6 de enero de 1975. En todo caso las modificaciones sugeridas por el Dr. Dóndoli no alteraban sustancialmente el proyecto y no incluían cambio alguno en el nombre del Centro. No obstante, a algunas personas (colegas y autoridades universitarias) les parecía muy "ambicioso" incluir la Física Planetaria en ese entonces. Debido a esto, primero se pensó en dejar como nombre Centro de Geofísica, pero después se decidió que era más apropiado Centro de Investigaciones Geofísicas. Además se consideró que el Centro debería formar parte de un instituto de investigación de la Facultad de Ciencias. Las justificaciones para la creación del Centro estaban contenidas en un documento titulado "Fundamentos para la creación de un Centro de Investigaciones Geofísicas en la Universidad de Costa Rica". El "Proyecto para la Creación del Centro de Investigaciones Geofísicas del Instituto de Ciencias" fue aprobado por el Consejo Asesor de la Facultad de Ciencias en su sesión No. 6, artículo II, del 15 de enero de 1975. Se declaró la aprobación del proyecto acuerdo firme y en el acta respectiva se lee: “...el señor $\mathrm{Ne}$ ville Clark agradece a los señores del Consejo por el voto unánime a favor del Centro y se pone a las órdenes de la Escuela de Geología para que juntos puedan lograr una mejor labor". En el proyecto aprobado, en la parte de los propósitos y objetivos del Centro de Investigaciones Geofísicas, el artículo 5 señalaba: "Promover un progreso constante en la investigación pura y aplicada en los siguientes campos: a) Geodesia y Gravimetría, b) Sismología, c) Física del Interior de la Tierra, d) Meteorología y Física Atmosférica, e) Oceanografía Física, f) Hidrología, g) Geomagnetismo y Aeronomía." (Se había suprimido "Física Planetaria y Espacial").

\section{LA IDEA DE INSTITUTO EN LUGAR DE CENTRO}

El Vicerrector de Investigación modificó el proyecto, comunicándole esto al Decano de la Facultad de Ciencias el 21 de marzo de 1975, en los siguientes términos: "Le envío copia del 
documento que le he presentado al señor Rector proponiendo la creación del Instituto de Geofísica. Me he permitido cambiar el proyecto original para adecuarlo a un machote general de reglamento de institutos que hemos aplicado al Instituto de Ciencias Sociales, al Instituto de Ciencias de la Salud y ahora al de ustedes. Hemos abandonado ya la idea de centros formando cinco grandes institutos. La idea la dejamos como una forma de coordinar programas, de promover seminarios, conferencias, etc..., pero ya no como una estructura administrativa. Por eso ustedes deben fundar un Instituto de Geofísica y otros que el futuro aconseje. El resto de los aspectos que contiene el proyecto de reglamento de ustedes lo pueden dejar como parte del reglamento interno de trabajo que lo propondrían luego de estar funcionando..." El documento enviado al Rector por el Vicerrector de Investigación fue analizado en la sesión No. 2139 del Consejo Universitario del 28 de abril de 1975, pero se pospuso su aprobación debido a que el Vicerrector de Investigación estaba fuera del país y se deseaba saber su opinión sobre lo que deben ser los institutos. ${ }^{2}$ Posteriormente, se volvió a la idea del Centro en lugar del Instituto. Así cuando, el 18 de julio de 1975, los profesores Walter Fernández, Luz Soltero y German Leandro le consultan al señor Vicerrector de Investigación, Dr. Guillermo Chaverri, sobre el estado en que se encuentra el proyecto para la creación del Centro de Investigaciones Geofísicas, éste les contesta el 22 de julio de 1975: "En respuesta a su estimable comunicación del 18 de julio pasado, tengo el agrado de contestar lo siguiente: El Centro de Geofísica con toda probabilidad formará parte del Instituto de Ciencias Exactas y Naturales (ICEN). Mientras tanto y para que no sufran atraso alguno las gestiones que ustedes deseen realizar, me permito autorizarles para realizar cualquier gestión de solicitud de fondos para el futuro Centro de Investigaciones Geofísicas (CIG) del ICEN. Para mayor validez legal de esta autorización, esta Vicerrectoría ha decidido establecer desde ahora un programa de Investigaciones Geofísicas con los fines y propósitos que se detallan en el proyecto de reglamento...".3

\section{NUEVA REVISIÓN DEL PROYECTO}

Al haber un cambio de Vicerrector de Investigación (el Dr. Manuel M. Murillo sustituyó al Dr. Guillermo Chaverri), los trámites para la aprobación de la creación del Centro de Investigaciones Geofísicas se hicieron más lentos. Por otro lado, el profesor Luis Diego Morales de la Escuela de Geología, estaba ahora al tanto del proyecto y participaba en las actividades de seguimiento del mismo. El 25 de mayo de 1976, el Dr. José Alberto Sáenz Renauld, Decano de la Facultad de Ciencias, le envió una carta al Dr. Manuel M. Murillo, Vicerrector de Investigación (con copia a los profesores Walter Fernández, Luis D. Morales, Neville Clark y César Dóndoli), donde le dice: "Desde hace más de un año profesores de las escuelas de Física y Geología de la Facultad de Ciencias, han venido gestionando la fundación del Centro de Geofísica, como parte del Instituto de Investigación de Ciencias Exactas y Naturales, ya consignado en el Estatuto Orgánico de la Universidad de Costa Rica... En la forma más atenta me permito solicitarle se sirva señalar los trámites pertinentes para llevar a cabo el establecimiento del indicado Centro". ${ }^{4}$

En la sesión No. 64 de la Escuela de Física, realizada el 15 de marzo de 1978, su Director (M.Sc. Neville Clark) informó sobre los trámites que se estaban haciendo en relación a la creación del Centro de Investigaciones Geofísicas. Mencionó que: "Algunos miembros de la Escuela de Física elaboraron el proyecto de reglamento respectivo y lo elevaron a la Decanatura. El señor Decano de ese entonces nombró una comisión con personal de las Escuelas de Geología y Física para su estudio. A raíz de este estudio se elaboró otro proyecto que fue enviado a la Vicerrectoría de Investigación, que ejecutó su propio proyecto con los arreglos pertinentes. Este documento fue conocido en la Asamblea de Escuela de Geología y a consecuencia de una protesta por la Dirección de la Escuela de Física, el señor Vicerrector de Investigación, Dr. Manuel Ma. Murillo, nombró una comisión integrada por los profesores: M.Sc. Armando Acuña Bonilla, Decano Facultad de Ciencias, Neville Clark, 
Director Escuela de Física, Dr. Javier Soley Alfaro y Dr Jorge Edo. Páez Portuguez, para que se discutiera nuevamente este documento. La Escuela de Física elaboró una serie de observaciones que fueron distribuidas a todos los miembros de la Asamblea (anexo 4) para su conocimiento". Las modificaciones sugeridas por la comisión mencionada fueron aprobadas en esa sesión. El anexo 4 mencionado era un "Proyecto de Reglamento del Instituto en Ciencias Exactas y Naturales" (IICEN), el cual había sido aprobado por la Vicerrectoría de Investigación, y las observaciones de la comisión nombrada por el Vicerrector de Investigación. ${ }^{5}$ En ese proyecto se incluían específicamente como parte del IICEN al Centro de Investigaciones en Productos Naturales y al Centro de Investigaciones Geofísicas, con sus respectivos reglamentos. La reglamentación del Centro de Investigaciones Geofísicas era muy general y en principio no excluía a ninguna de las ciencias geofísicas.

La Escuela de Geología había presentado modificar el nombre de Centro de Investigaciones Geofísicas por Centro de Investigaciones en Ciencias Geológicas y Físicas a lo cual la Escuela de Física se opuso argumentando: 6 "La modificación que propone la Escuela de Geología, de que este Centro se llame de Ciencias Geológicas y Físicas, parece tener alcances muy amplios y desvirtuar uno de los principales objetivos cual es el de estimular a grupos de investigadores a trabajar coordinadamente en campos definidos. Si se aceptase el nombre que propone Geología, habría que adscribir a todos los investigadores que actualmente son profesores de las Escuelas de Geología y Física, y prácticamente la situación no cambiaría. El nombre de Centro de Investigaciones Geofísicas nos parece más apropiado". Además, la Escuela de Geología sugirió un transitorio I que decía: "El Centro de Investigaciones Geofísicas se dedicará preferentemente a continuar las investigaciones sismológicas en curso, aumentándolas y extendiéndolas -de acuerdo con las posibilidades económicas- con más estaciones de observación, y a coordinar las investigaciones vulcanológicas y geomagnéticas que ahoran realizan las escuelas de Geología y Física" [6]. La Escuela de Física sugirió que se eliminara ese transitorio o "...si no se quiere eliminar, que se modifique en cuanto a su redacción para que realmente sea un transitorio y no una cláusula permanente". La sugerencia de incluir ese transitorio dejaba de lado el proyecto aprobado por unanimidad en el Consejo Asesor de la Facultad de Ciencias en su sesión No. 6 del 15 de enero de 1975, donde se incluían claramente todas las disciplinas geofísicas, incluyendo la Meteorología y la Oceanografía Física. Además, dicho transitorio no concordaba con el cambio de nombre propuesto por la Escuela de Geología. Cuando los profesores de la Escuela de Geología propusieron ese transitorio, los profesores Walter Fernández y Jorge Amador se encontraban realizando sus estudios de doctorado (ambos en Ciencias Atmosféricas) en Inglaterra, y el Profesor Federico Güendel, otro colega de Física interesado en el tema, se encontraba en Estados Unidos, donde estuvo hasta junio de 1978 especializándose en Geofísica de la Tierra Sólida. Ellos consideraban que el Centro debía incluir todas las ciencias geofísicas en que se hiciera investigación. Por otro lado, con Oceanografía Física existía el inconveniente de que el Dr. Manuel M. Murillo, Vicerrector de Investigación en ese entonces, estaba interesado en crear un Centro de Investigación en Ciencias del Mar y Limnología. Aunque éste se gestó después del Centro de Investigaciones Geofísicas, se creó antes. ${ }^{7}$

\section{APROBACIÓN DE LA CREACIÓN DEL CENTRO}

En el proyecto de reglamento del IICEN el transitorio II decía: "La designación del Instituto de Investigaciones en Ciencias Exactas y Naturales y la instalación del Consejo Asesor del Instituto no se realizarán sino pasados dos años a partir del momento en que este reglamento sea promulgado por el Consejo Universitario". Durante ese período los Centros de Investigaciones en Productos Naturales y en Geofísica funcionarán como unidades dependientes administrativamente de la Vicerrectoría de Investigación, esto con el propósito de facilitar la consolidación de los 
dos Centros de Investigación que, posteriormente, se integrarán al Instituto de Investigaciones en Ciencias Exactas y Naturales". Con base en este transitorio y tomando en consideración las observaciones mencionadas, el Vicerrector de Investigación tramitó ante el Consejo Universitario el proyecto de reglamento del Centro. El Centro de Investigaciones Geofísicas (CIGEFI) fue creado el 5 de febrero de 1979, cuando el Consejo Universitario en su sesión No. 2538, artículo 19, aprobó su reglamento. Su primer Consejo Científico fue instalado el 4 de junio de 1979, con los siguientes investigadores: Federico Güendel, de la Escuela de Física, Jorge A. Gutiérrez, de la Escuela de Ingeniería Civil, y Rodolfo Madrigal, Siegried Kussmaul, Walter Montero, Sergio Paniagua, Francois Rivier y Carlos Torres, de la Escuela de Geología. Esta sesión fue presidida por el Vicerrector de Investigación, Dr. Manuel M. Murillo. Asistieron también, el Decano de la Facultad de Ingeniería (Ing. Rodrigo Orozco), el Decano de la Facultad de Ciencias (Dr. Armando Acuña) y la Directora de la Escuela de Física (M.Sc. Luz María Moya). El M.Sc. Rodolfo Madrigal participó en su doble condición de investigador y de Director de la Escuela de Geología. Posteriormente, por encargo del Vicerrector de Investigación, el M.Sc. Walter Montero actuó como Coordinador del CIGEFI mientras se tramitaba el nombramiento del Director del Centro. A principios de 1981, se incorporaron al CIGEFI Jorge A. Amador y Walter Fernández, luego de haber concluido sus estudios de doctorado en Inglaterra y haber retornado a la Universidad.

En el primer semestre de 1981, siendo el M.Sc. Walter Montero Coordinador del CIGEFI, se realizaron varias sesiones del Consejo Científico. En estas sesiones, se trataron, además de otros temas, aspectos relacionados con la planificación de la construcción del CIGEFI y con el programa de investigación del Centro, propuestas de incorporación de nuevos miembros al Consejo Científico y nombramiento de la terna para el puesto de Director.

\section{DIVULGACIÓN E INICIO SISTEMÁTICO DE LAS PRIMERAS ACTIVIDADES DEL CENTRO}

En 1980 se publicó un artículo en Prociencia [8], en el cual se describían las principales actividades que se realizaban en ciencias geofísicas en la Universidad de Costa Rica y se informaba de la creación del Centro de Investigaciones Geofísicas. En febrero de 1981 se publicó en el periódico La Nación un reportaje sobre el Centro de Investigaciones Geofísicas, 9 titulado "Geofísica: Una ayuda en la prevención de los desastres naturales", donde se informaba sobre la creación del Centro y se describían las actividades en Vulcanología, Sismología y Física Atmosférica, enfatizando su importancia en la prevención de los desastres naturales. Este reportaje fue comentado días después en el Eco Católico ${ }^{10}$ donde, bajo el título de "Comentando la noticia: Prevención de los desastres naturales", se concluía: "El reconocimiento de los dones que hemos recibido del creador tiene lugar cuando esfuerzos colectivos, como el que aparece en esta noticia, empiezan a realizarse".

Aunque el Centro se creó en 1979 no fue hasta 1981 que se nombró su primer director, el M.Sc. Luis Diego Morales. Al hacerse esto, se iniciaron de una forma sistemática sus diferentes actividades científicas. Posteriormente lo sustituyeron en el cargo: Dr. Walter Fernández (19831987), Dr. Jorge A. Amador (1988-1993), Dr. Francisco Javier Soley (1993-1997) y Dr. Jorge A. Amador (1997-). Actualmente, el Centro se encuentra desarrollado y cuenta con edificio propio. La labor de sus investigadores se proyecta a la comunidad científica nacional e internacional.

\section{COMENTARIO FINAL}

El objetivo de este trabajo ha sido solamente describir los antecedentes de la creación del Centro de Investigaciones Geofísicas (CIGEFI). Debido a esto, no se ha incluido información detallada sobre las actividades del centro, luego de su creación. 
Cabe destacar que los aspectos aquí mencionados sobre los antecedentes de la creación del Centro de Investigaciones Geofísicas, no fueron descritos en otros trabajos publicados sobre el tema. ${ }^{11}$

\section{AGRADECIMIENTOS}

El autor está agradecido con el Dr. Federico Güendel, M.Sc. German Leandro, M.Sc. Walter Montero y M.Sc. Sergio Paniagua por sus valiosos comentarios y sugerencias.

\section{REFERENCIAS BIBLIOGRÁFICAS Y NOTAS}

1 Oficio VI-205-75 del 21 de marzo de 1975.

2 Oficio DFC-172-75 del 22 de mayo de 1975 y Oficio VI-305-75 del 3 de junio de 1975.

3 Oficio VI-475-75 del 22 de julio de 1975.

4 Oficio DFC-213-76 del 25 de mayo de 1976.

5 Varios oficios se refieren a las objeciones de la Escuela de Física: EF-066-78 del 13 de febrero de 1978, VI-304-78 del 13 de febrero de
1978, EF-021-78 del 27 de marzo de 1978, DFC-105-78 del 28 de marzo de 1978.

Armando Acuña Bonilla, Neville Clark, Javier Soley Alfaro y Jorge Edo. Páez Portuguez. "Observaciones a los proyectos de la Vicerrectoría de Investigación y de la Escuela de Geología sobre el Instituto de Ciencias Exactas y Naturales". Propuesta por la comisión ad.hoc a la Asamblea de la Escuela de Física No. 64 del 15 de marzo de 1978.

$7 \quad$ El Vicerrector de Investigación en ese entonces era el Dr. Manuel M. Murillo, cuya especialidad es Biología Marina.

8 W. Fernández. "Las ciencias geofísicas en la Universidad de Costa Rica”. Prociencia, No. 25, pág. 10 (1980).

9 Fabián Barrantes. "Geofísica: Una ayuda en la prevención de los desastres naturales”. La Nación, lunes 9 de febrero de 1981, pág. 1B.

10 "Comentando la noticia: Prevensión de desastres naturales". Suplemento Betania del Eco Católico, Domingo 22 de febrero de 1981, pág. 2 del suplemento.

11 Luis Diego Morales. "Historia del desarrollo de las disciplinas geofísicas en Costa Rica”. Editorial Tecnológica de Costa Rica, Cartago, Costa Rica, págs. 256-272 (1989). 